\title{
Theoretical and experimental study on vibration modal for simply supported bridge
}

\author{
Ying Wang ${ }^{1}$, Li Peng ${ }^{1}$, Xuewei Zhang ${ }^{1}$, Lingfeng Chen ${ }^{1}$, Tao Chen ${ }^{1}$, and Congqi Fang *1 \\ ${ }^{1}$ College of civil engineering, Shanghai Normal University, Shanghai 201418, China
}

\begin{abstract}
For the highway bridges with small or medium span, a vehicle will stay on the bridge after the excitation. So the measured values of the vibration characteristics of the bridge are related to the vehiclebridge system. In order to analyse the natural vibration characteristics of simply supported bridge and the loaded frequency of vehicle-bridge system, theoretical analysis was carried out firstly, then the simply supported beam calculation model was built based on finite element method, and the results were compared with the measured values. The finite element model of a mass block-simply supported beam system was built by regarding the vehicles as a concentrated mass block attached. Both the vibration modals of the bridge system and the loaded frequencies of the vehicle-bridge system under diffident vehicle positions were obtained by software analysing. As a result, the variation characteristics of the loaded frequency for this simply supported bridge were extracted and discussed through the comparison of the results from both finite element method and the measured.
\end{abstract}

\section{Introduction}

With the sustainable development of national economy and engineering technology, achievements of bridge construction change with each passing day. The amount of existing bridges is increasing rapidly since a large number of newly built bridges were putting into operation. In recent years, the on-site testing technology is used widely in bridge test and evaluation. As an onsite test for highway bridges with short or medium span, their vibrations were excited usually by a single vehicle, and the vehicle will stay on the bridge after excitation, so the measured values of the bridge under the action of a single vehicle are the vibration characteristics of vehiclebridge system. In other words, the measured frequencies are the loaded frequencies of the vibration system. The literatures show that the frequency differences between the vehicle-bridge system and bridge structure cannot be neglected [1], of even larger [2] than the changes of structural damage.

In this paper, the authors firstly analysed a simply supported steel beam theoretically, then a finite element model was built based on the software of Ansys, and the analysis results were compared with the measured values. Next, the finite element model of a mass block-simply supported beam system was built by regarding the vehicles as a concentrated mass block attached. At last, the analysis results from software were compared with the tested values, and the authors gave some useful conclusions which are appreciated for reference.

\section{Profile of the simply supported beam}

The simply supported steel beam with a span of $690 \mathrm{~mm}$ between two points, and with a rectangular cross section of $50 \mathrm{~mm}$ (width) $\times 8.5 \mathrm{~mm}$ (height) is shown in Fig. 1 . The elastic modulus and Poisson ratio of the steel beam are $2.06 \times 10^{5} \mathrm{MPa}$ and 0.3 respectively. Furthermore, the mass density of steel is $7850 \mathrm{~kg} / \mathrm{m}^{3}$.

\section{Modal frequency analyses}

\subsection{Theoretical analysis}

As a simply supported beam with uniform section, its natural frequencies can be described as following [3]:

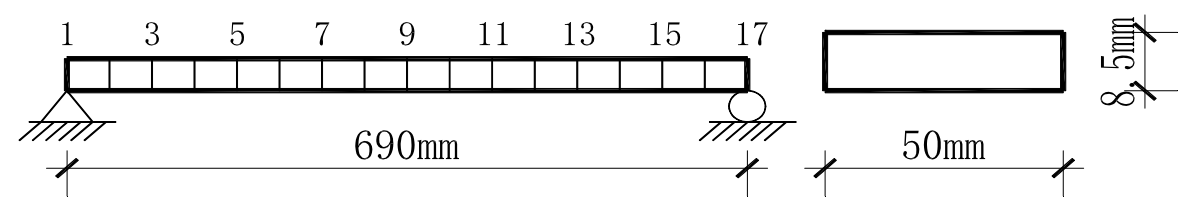

Fig.1. Sketch of a simply supported steel beam 


$$
f_{n}=n^{2} \pi^{2} \sqrt{\frac{E I}{m L^{4}}} \quad \mathrm{n}=1,2,3, \cdots(1)
$$

Where $f_{\mathrm{n}}$ is the frequency of each order, EI is the flexural stiffness of cross section, $\mathrm{m}$ is mass for per unit length, and $\mathrm{L}$ is the span between two support points.

According to the physical property parameters and size parameters of the steel beam described as above, the first 5 order natural vibration frequencies can be calculated as in Table 1.

Table 1 The first 5 order natural frequency from

\begin{tabular}{|c|c||c|c|}
\multicolumn{4}{c|}{ theoretical analysis $(\mathrm{Hz})$} \\
\hline order & frequency & order & frequency \\
\hline 1 & 41.449 & 2 & 165.797 \\
\hline 3 & 373.044 & 4 & 663.189 \\
\hline 5 & 1036.225 & & \\
\hline
\end{tabular}

\subsection{Finite element analyses}

The finite element model using commercial software Ansys [4-5] was built as shown in Fig.2. The conventional beam element was used to simulate the steel beam, and block Lanczos algorithm was adopted for modal extraction.

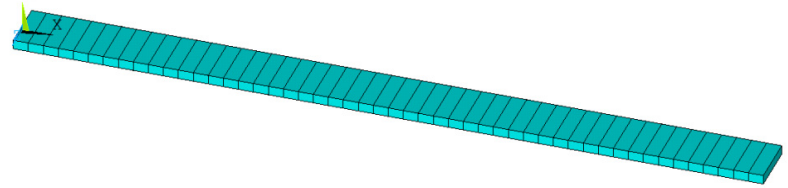

Fig.2 Finite element model for steel beam

The vibration modal parameters can be obtained by running Ansys modal analysis module. The first 5 order natural vibration frequencies are showing in Table 2. And the related vibration deformation diagrams are showing in Fig.3.

Table 2 The first 5 order natural frequency from Ansys

\begin{tabular}{|c|c||c|c|}
\hline order & frequency & order & frequency \\
\hline 1 & 41.469 & 2 & 165.840 \\
\hline 3 & 373.030 & 4 & 663.880 \\
\hline 5 & 1035.200 & & \\
\hline
\end{tabular}
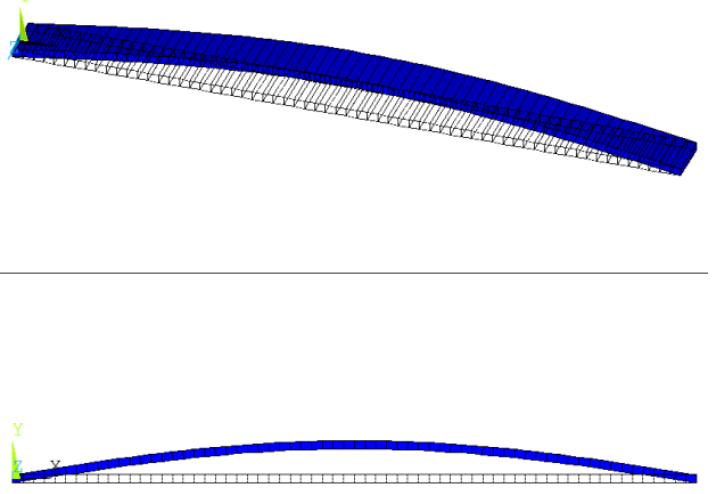

(a) The $1^{\text {st }}$ order
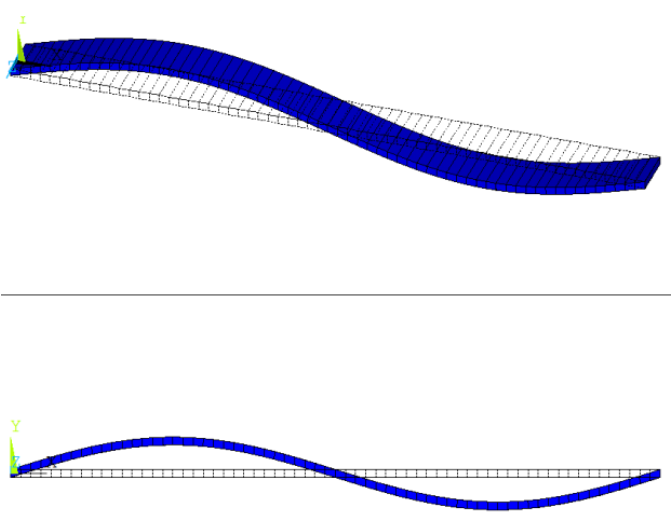

(b) The $2^{\text {nd }}$ order
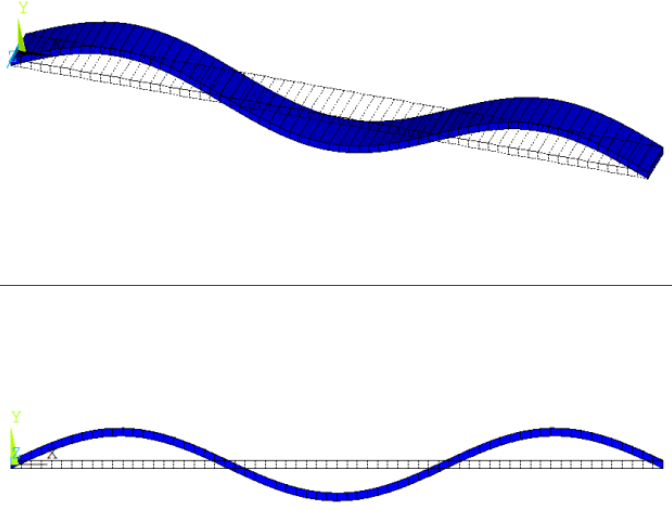

(c) The $3^{\text {rd }}$ order
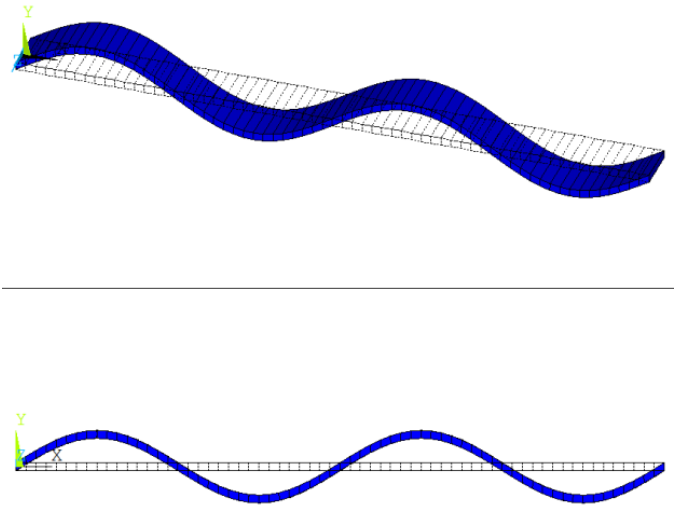

(d) The $4^{\text {th }}$ order
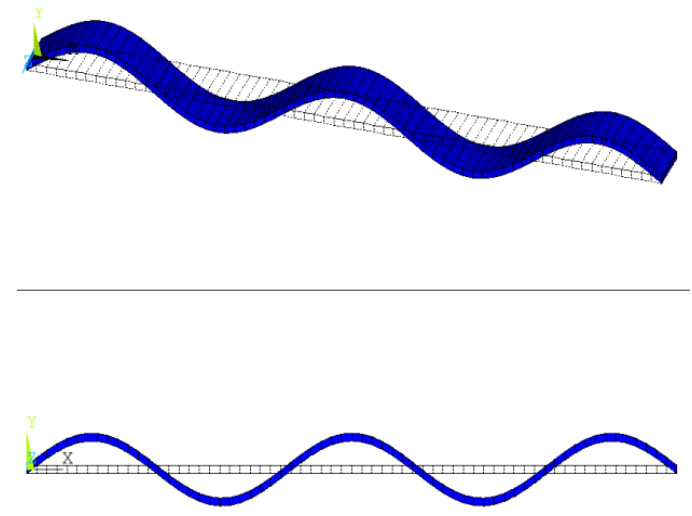

(e) The $5^{\text {th }}$ order

Fig.3. The first 5 order vibration deformation diagram 


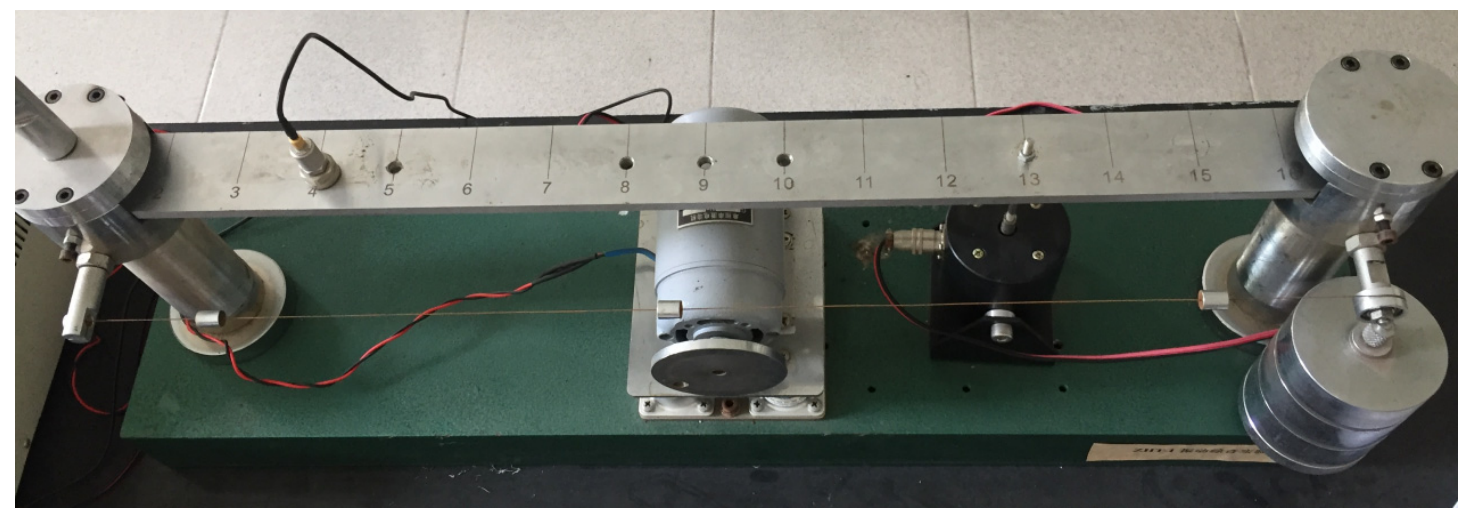

Fig.4. The forced vibration test for steel beam

\subsection{Experimental test}

The ZHT-1 integrated experiment teaching system was adopted for vibration test. The size and the material parameters are the same with the Ansys model mentioned above. In this paper, the natural frequencies of the simply supported steel beam were obtained by free vibration and forced vibration methods. The test results were the mean values of the both methods, showing in Table 3. And Fig.4 is the picture of forced vibration test.

Table 3 The first 5 order natural frequency from test $(\mathrm{Hz})$

\begin{tabular}{|c|c||c|c|}
\hline order & frequency & order & frequency \\
\hline 1 & 41.653 & 2 & 167.009 \\
\hline 3 & 371.523 & 4 & 657.912 \\
\hline 5 & 1028.969 & & \\
\hline
\end{tabular}

\subsection{Results analysis}

Three approaches are involved in carrying out the natural frequencies of the simply supported steel beam. The results are showing in Table 1 to 3 . Supposing the experimental test results are the real values, the relative errors were calculated shown in Table4.

Table 4 Relative error for natural frequency（\%)

\begin{tabular}{|c|c|c|}
\hline order & $\begin{array}{c}\text { Finite element } \\
\text { method }\end{array}$ & $\begin{array}{c}\text { Theoretical } \\
\text { analysis method }\end{array}$ \\
\hline 1 & 0.441744892 & 0.489760641 \\
\hline 2 & 0.699962277 & 0.725709393 \\
\hline 3 & 0.405627646 & 0.409395919 \\
\hline 4 & 0.755116186 & 0.802082953 \\
\hline 5 & 0.605557602 & 0.705171876 \\
\hline
\end{tabular}

From Table 4, it shows that the relative errors are all less than $1 \%$ for both methods. Furthermore, the errors from finite element method are less than theoretical analysis, it means, the natural frequencies from finite element method coincide well with test results. So the finite element method using beam element from Ansys can be adopted to simulate the steel beam vibration.

\section{Loaded frequency analyses}

\subsection{Finite element method}

The finite element model of a mass block-simply supported beam system was built by regarding the vehicles as a concentrated mass block attached. For convenience of comparing the results from experimental tests, the simply supported steel beam was divided in 16 equal parts, including 17 notes, as shown in Fig.1. It's 40.295 gram for each mass block. For simulating the passing vehicles on the bridge, the position of the mass block changed along the length of the beam. The loaded frequencies of the mass block-beam system were carried out by running the Ansys software. The first 5 order of loaded frequencies are showing as Fig. 5.
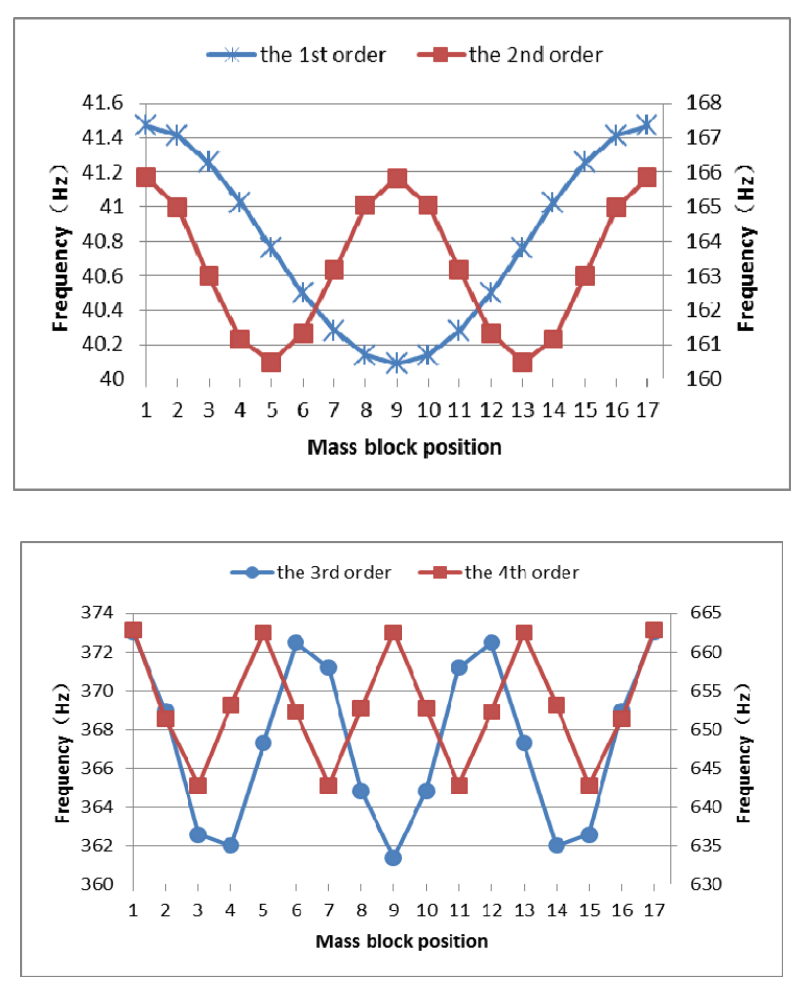


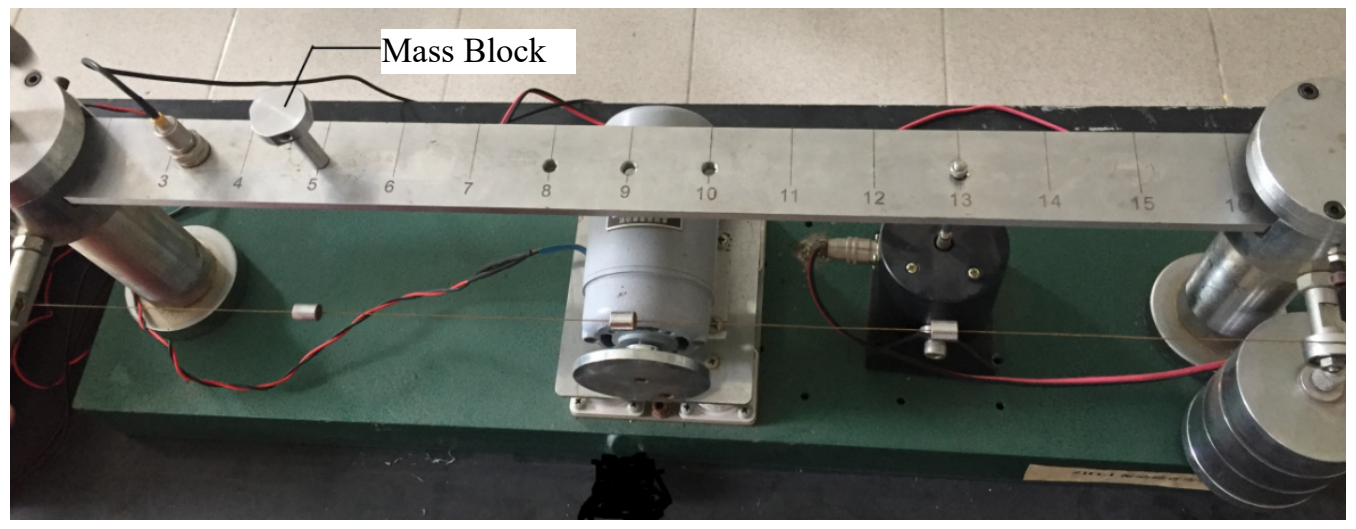

Fig.6. Mass block-simply supported beam system

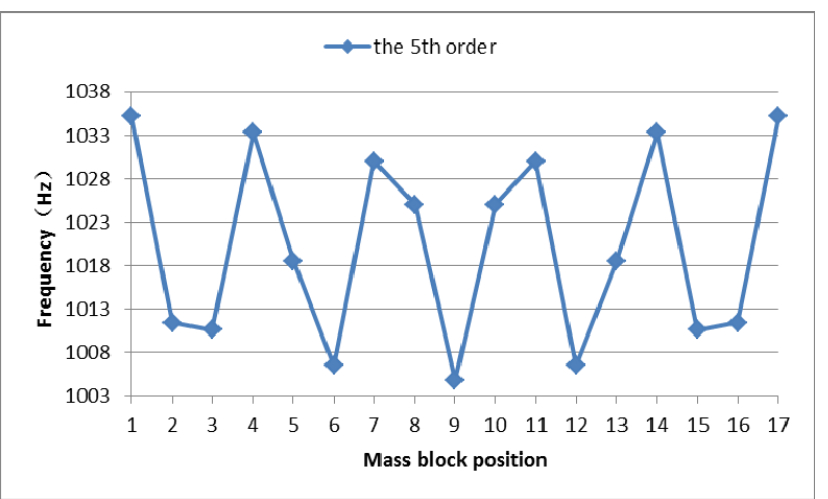

Fig.5. The loaded frequency of mass block-beam system

\subsection{Experimental test}

The ZHT-1 integrated experiment teaching system was adopted for vibration test again. The 80.59 gram mass block was fixed at note No.5 firstly, then at No. 8, No. 9 and No. 10 at last in order to simulate the passing vehicle on the bridge. Fig. 6 is the picture of vibration testing when the mass block is at note No.5.

The natural frequencies of the mass block-simply supported steel beam system were obtained by free vibration and forced vibration methods respectively. The test results were the mean values of both methods, showing in Table 5.

Table 5 . The first 5 order natural frequency for mass block-beam system from test $(\mathrm{Hz})$

\begin{tabular}{|c|c|c|c|c|}
\hline $\begin{array}{c}\text { order } \\
\text { Note }\end{array}$ & 5 & 8 & 9 & 10 \\
\hline 1 & 41.079 & 40.760 & 40.150 & 40.680 \\
\hline 2 & 162.323 & 165.250 & 165.810 & 165.240 \\
\hline 3 & 369.380 & 365.200 & 364.180 & 365.210 \\
\hline 4 & 662.790 & 656.260 & 662.390 & 656.240 \\
\hline 5 & 1023.540 & 1027.92 & 1007.460 & 1027.910 \\
\hline
\end{tabular}

\subsection{Loaded frequency results analysis}

\subsubsection{Finite element results analysis}

Both the loaded frequencies of the mass block-beam system and the natural frequencies of the steel beam are obtained by Ansys software analysis. The relative errors of the two kinds of frequencies can be calculated and shown in Fig. 7.

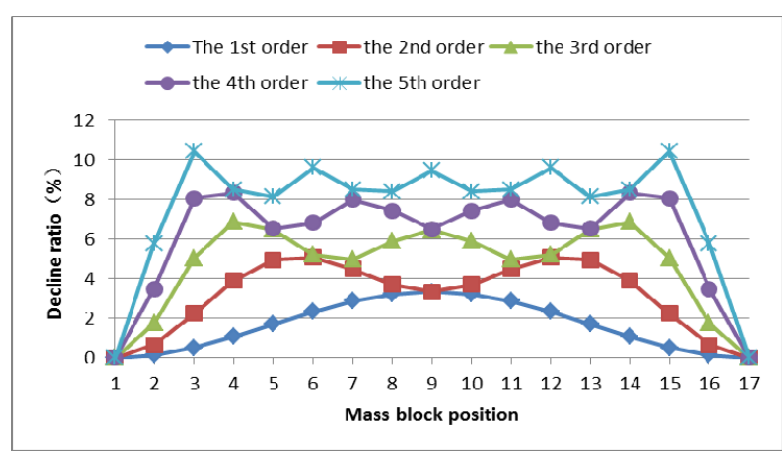

Fig.7. Relative error for loaded frequency by Ansys

\subsubsection{Test results analysis}

Regarding the limited positions can fix the mass block on the steel beam, four different positions carried out for loaded frequency testing. Comparison the loaded frequencies of the mass block-beam system with the natural frequencies of the steel beam, the relative errors can obtain, showing in Table 6 .

Table 6 . The relative error of loaded frequency for test

\begin{tabular}{|c|c|c|c|c|c|c|c|c|}
\hline \multirow{2}{*}{$\begin{array}{c}\text { Vibration } \\
\text { order }\end{array}$} & \multicolumn{2}{|c|}{ Note NO.5 } & \multicolumn{2}{c|}{ Note NO.8 } & \multicolumn{2}{|c|}{ Note NO.9 } & \multicolumn{2}{|c|}{ Note NO.10 } \\
\cline { 2 - 9 } & Ansys & Test & Ansys & Test & Ansys & Test & Ansys & Test \\
\hline 1 & 1.71 & 0.94 & 3.21 & 1.71 & 3.33 & 3.18 & 3.21 & 1.90 \\
\hline 2 & 3.23 & 2.12 & 0.49 & 0.36 & 0.01 & 0.02 & 0.49 & 0.36 \\
\hline 3 & 1.54 & 0.98 & 2.20 & 2.10 & 3.13 & 2.37 & 2.20 & 2.10 \\
\hline 4 & 0.05 & 0.01 & 1.54 & 1.00 & 0.05 & 0.07 & 1.54 & 1.00 \\
\hline 5 & 1.61 & 1.13 & 0.99 & 0.70 & 2.94 & 2.68 & 0.99 & 0.70 \\
\hline
\end{tabular}

\section{Discuss and conclusions}

In this paper, the authors selected the simply supported beam as research object. Firstly, the theoretically analysis was carried out for natural frequencies, and then the finite element model was built using Ansys. By regarding the vehicle on the bridge as mass block, the finite element model was built for mass block-beam system as well. And the comparison of the loaded frequencies of the mass block-beam system with the natural frequencies of the beam was carried out. 
The following conclusions can be drawn from the analysis above:

(1)The loaded frequencies are slightly less than the natural frequencies of the simply supported beam. Fig 7 shows that the relative error drops of each mass block position except two ends. The same conclusion can be drawn from the theoretical analysis equation (1).

(2)Curve shape of the relative error for loaded frequency of each vibration order is similar with the vibration deformation diagram. For example, the doublewave curve shape of the 2nd order in Fig 7 is the similar with the 2nd order of Fig5.

(3)The position of the vehicle on the bridge has influence on the loaded frequencies. From Fig7, it shows that the relative error is larger than other position when the mass block is on the position of maximum amplitude. In other words, the position of maximum amplitude is the most sensitive position for loaded frequency.

(4)The beam element of Ansys can be adopted to simulate the vibration characteristics of simply supported bridge. Both Table 4 and 6 show that the frequency results from Ansys are very close to the results from experimental test. So the beam element from Ansys can be used to simulate the loaded frequency of vehiclebridge system.

\section{Acknowledgment}

The authors are grateful to Mr. Cui Qi who has offered selfless support on experimental tests.

This paper was supported partially by the project from Shanghai Science and Technology Committee (NO. 18070502900).

\section{References}

1. Ying Wang, Li Peng. Research on vertical loaded frequency for haif-through arch brisge. Structural Engineers. Vol.35, No.4: 37-41 ( 2019)

2. Yongchun Chen, Guojin Tan, Hanbing Liu, et al. Test frequencies freely supported beam of highway bridge under effect of vehicles. Journal of Jilin University (Engineering and Technology Edition). Vol. 39, No.6: 1492-1496 (2009)

3. Ray W. Clough, Joseph Penzien. Dynamics of structures (Second edition). Computers and Structures, Inc. California (2003).

4. Xinmin Wang. Structural Dynamic Analysis and Application with ANSYS. China Communication Press Co., Ltd. (2014)

5. Ying Wang, Youting Li, Li Peng, etc. An analysis of irregular rigid-frame bridge's responses to the lateral vibration caused by moving vehicular loads. Journal of Shanghai Normal University (Natural Sciences). Vol.45, No.1: 103-107 (2016). 